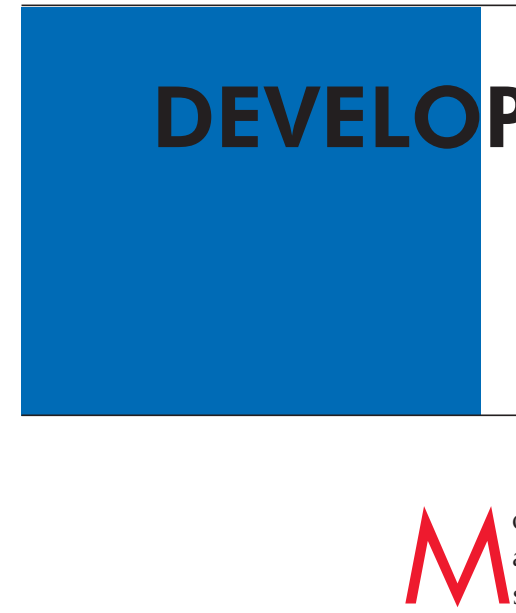

\title{
PMENT AND STRUCTURE OF THE ATRIAL SEPTUM
}

Robert H Anderson, Nigel A Brown, Sandra Webb

Heart 2002;88:104-110 of cardiac embryology. These illustrate the formation of primary and secondary atrial septums as overlapping muscular sheets that grow into the common atrium. This type of illustration implies that similar morphological mechanisms of development lead to the formation of these two "septums". This is not so. To the best of our knowledge, there is no evidence existing which supports this concept of growth of a second muscular shelf into the developing atriums so as to overlap the primary atrial septum, and to provide the rims of the definitive oval fossa. On the contrary, it has long been established ${ }^{2}$ that the superior border of the "septum secundum", in other words the superior rim of the oval fossa, is an infolding of the atrial roof. In this respect, case reports are to be found that describe the formation of lipomas within the supposed "septum secundum". ${ }^{3}$ Careful study of such lipomas, ${ }^{4}$ along with scrutiny of the published images, ${ }^{3}$ reveals that the fat accumulates within the deeply infolded superior interatrial groove.

All the "classical" accounts of atrial septal development have also ignored totally the contribution to atrial septation made by the "spina vestibuli", a structure first described by His in the 19th century. Similarly, they take no account of the contributions made by the mesenchymal cap which clothes the leading edge of the muscular primary atrial septum. ${ }^{6}$ In reality, therefore, more structures contribute to division of the atriums than the so-called primary and secondary septums. ${ }^{7}$ It is appreciation of the roles of all these various components that provides the basis for understanding the proper structure of the muscular walls interposed between the right and left atriums. ${ }^{8}$ This information, in this burgeoning era of interventional catheterisation, is now of major practical importance to the cardiologist. ${ }^{9}$ In this review, we will describe all the building blocks that contribute to the walls that separate the definitive right and left atrial chambers, showing how knowledge of their position provides the basis for understanding the definitive anatomy.

\section{DEVELOPMENT OF THE ATRIAL SEPTUM}

During early development, the initially tubular heart is suspended along its length by a dorsal mesocardium. It is at this stage that the so-called "straight tube" can be recognised, albeit that it has been shown that, with time, this part of the tube gives rise only to the ventricular component of the definitive heart. ${ }^{10}{ }^{11}$ At the stage when the developing heart tube is straight, the outflow tract, the atrioventricular canal, and the primary atrium have all still to form.

With continued growth, the ventricular component of the tube liberates itself from the body wall in the process called looping, thus losing most of its dorsal mesocardial connection. Concomitant with looping, there is expansion of the caudal inlet, part of the tube to form the primary atrium. At the same time, there is recruitment of extracardiac cells to expand the outlet portion of the tube at the arterial pole. ${ }^{11}$ The newly formed atrial component is directly continuous with the systemic venous tributaries. These wide venous channels, which carry the blood back to the heart from both the right and left sides of the body, are from the outset connected directly to the primary atrial component. In man, however, from the earliest stages we have been able to study, there is a pronounced asymmetry in the connections of the right and left sided venous channels to the heart. From the outset, the left sinus horn is incorporated within the developing left atrioventricular junction as it extends to terminate in the atrium. Despite the asymmetry, at this stage the primary atrial component initially receives large systemic venous tributaries from both sides of the embryo (fig l). It communicates with the ventricular loop through the atrioventricular canal. Towards the developing spinal column, the atrial cavity, now receiving the systemic venous tributaries, abuts directly on the body wall. In this area, the myocardial and mesenchymal tissues retain their continuity, persisting as a dorsal mesocardium (fig 1A). This connection between heart and body will, eventually, provide the portal of entry for the pulmonary vein. ${ }^{6}$ At this stage, however, the lungs are only just starting to bud from the trachea. Also, at this stage, the endothelium lining the heart tube separates the lumen of the atrium from the mesenchyme of the body wall (fig 1B). In the human, 

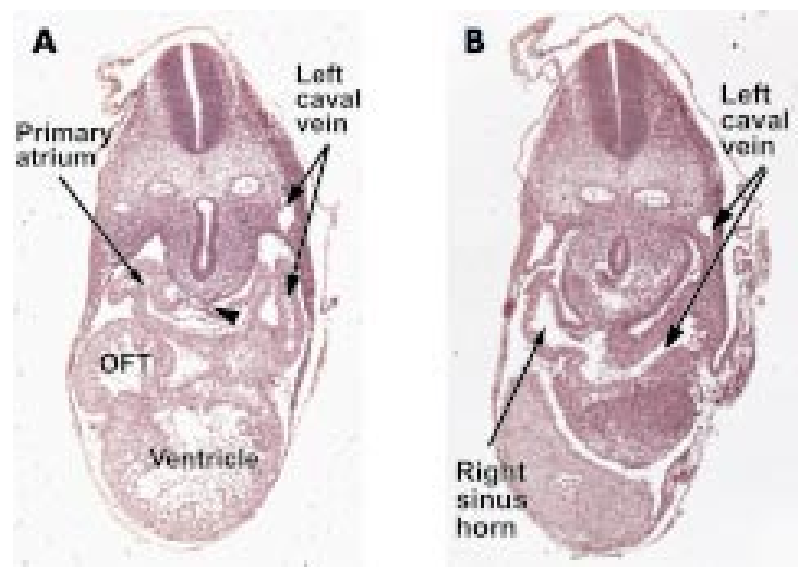

Figure 1 These sections are taken from a human embryo at Carnegie stage 12, sectioned in the short axis of the body. Section A shows how the walls of the primary atrium are continuous with the body of the embryo through the so-called "dorsal mesocardium" (arrowhead). Section B shows the continuity between the primary atrial component and the sinus horns, which are asymmetrical as they join the heart, the left horn being continuous with the left cardinal vein, which becomes the left superior caval vein.

this stage of development is reached when the entire embryo is less than $5 \mathrm{~mm}$ long. This is in the sixth week of development, and is representative of the 12th horizon within the temporal sequence established from the Carnegie collection of embryos. ${ }^{12}$

The primary atrium at this early stage is a common cavity. As a prelude to division of the cardiac cavities by formation of muscular septums, endocardial cushions have already formed within the atrioventricular canal, and also within the outflow tract which leads from the ventricular loop to supply the arteries of the developing branchial arches. As already shown (fig 1), initially the systemic venous tributaries join with the primary atrium from both sides of the embryo. These channels are the horns of the systemic venous sinus, the "sinus venosus", or the ducts of Cuvier. At this early stage, there are no discrete anatomical landmarks that mark boundaries between the venous horns and the primary atrium. Furthermore, at this stage, as also explained, the lung buds are only just starting to develop, and the pulmonary vein has yet to appear. The left sinus horn diminishes in size through and beyond this period, becoming more fully incorporated into the left atrioventricular junction as the coronary sinus. Throughout its development, the coronary sinus, and its precursor, the left sinus horn, possesses its own discrete walls (figs l and 2). These walls form a discrete bifurcation with the right sinus horn at their junction with the developing right atrium, which persist as the part of the right atrium called the "sinus septum".

Subsequent to rearrangements in the structure of the systemic venous tributaries, the entire systemic venous compartment comes to drain exclusively to the right side of the primary atrium. As it does so, valve-like structures are produced at the right and left borders of its junction with the primary atrial component of the heart tube (fig 2). The appearance of these valves permits, for the first time, the boundaries of the systemic venous sinus to be located with certainty relative to the other parts of the developing atriums. While the systemic venous component has been establishing its own identity in this fashion, further important changes have occurred in the primary atrial segment. Expansions to right and left have formed the atrial appendages (fig 2A); concomitant with development of the lungs in the body wall behind the heart, a venous channel, the primary pulmonary vein canalises within the dorsal mesocardium (fig 2C). Canalisation of this channel brings the pulmonary venous plexuses into continuity with the cavity of the developing left atrium. Initially, a solitary pulmonary venous channel enters the left atrial part of the primary atrial component inferiorly and posteriorly, the entrance being bounded by two ridges which demarcate the site of the persisting dorsal mesocardium. The right of these two ridges becomes particularly prominent (fig 3). It is this structure that $\mathrm{His}^{5}$ nominated as the "spina vestibuli".

All of these changes and remodellings relative to the primary atrium set the scene for atrial septation. The first indication of this septation is the formation of the primary atrial septum, first seen as a muscular crescent in the atrial roof. As it grows into the atrial cavity, it carries a mesenchymal cap on its leading edge (fig 2B). With continued growth, septum and cap move towards the atrioventricular endocardial cushions which, at the same time, are dividing the atrioventricular canal. The space between the mesenchymal cap on the leading edge of the primary atrial septum and the fusing atrioventricular cushions is the primary atrial foramen.

The primary septum, from the outset, is continuous inferiorly with the right pulmonary ridge, now prominent as the vestibular spine (fig 3). As it grows into the primary atrial
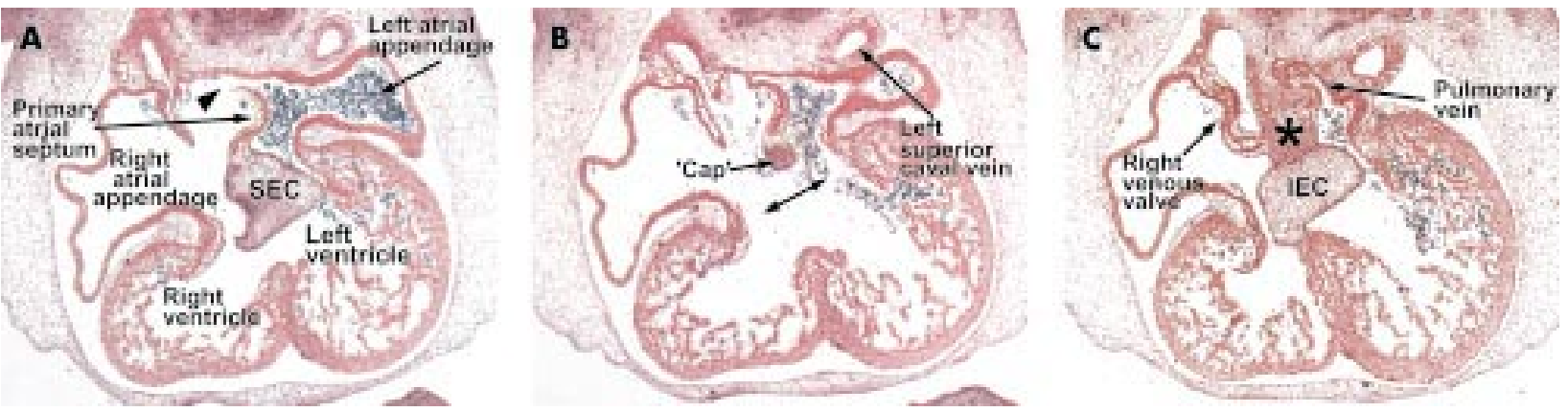

Figure 2 These three sections are from a human embryo at Carnegie stage 16, and are cut in the frontal plane of the heart. Section $\mathrm{A}$ is through the superior atrioventricular endocardial cushion (SEC). Note that the upper end of the primary septum has broken down to form the secondary foramen (arrowhead). The endocardial cushions have yet to fuse at this stage, and section B is taken between the cushions. Note the mesenchymal cap on the leading edge of the primary septum. Section $C$ is through the inferior cushion (IEC) and shows how tissue from the body of the embryo enters the heart through the vestibular spine (asterisk). Note the solitary pulmonary vein joining the left side of the primary atrium. 

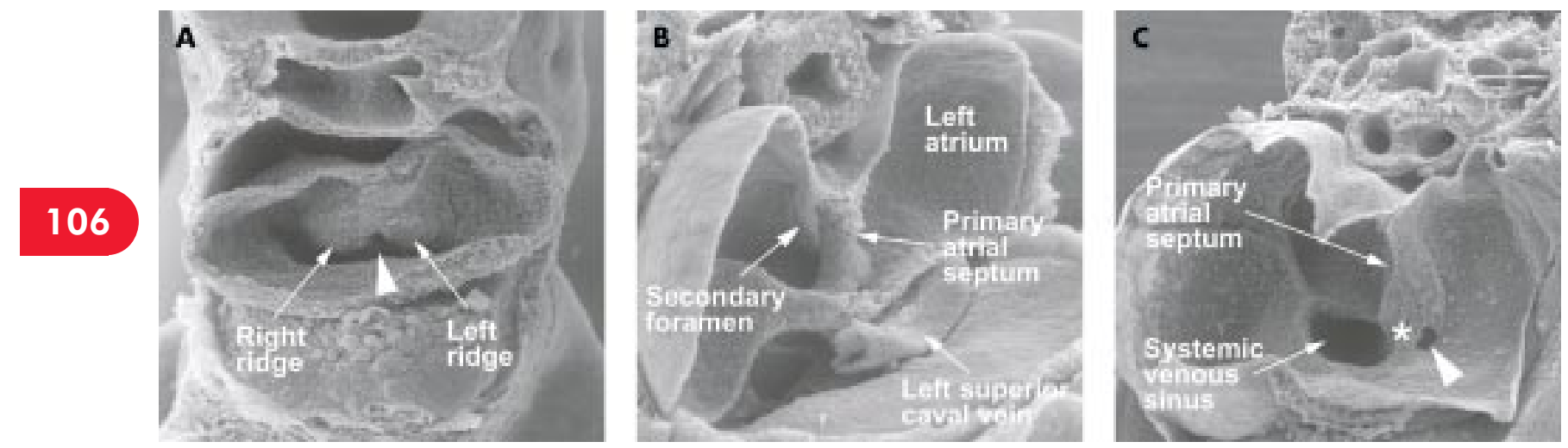

Figure 3 These pictures are scanning electron micrographs of the developing mouse heart. Picture A, from a mouse at embryonic day 9.5, with 28 somites, shows the stage before canalisation of the pulmonary vein. When formed, the vein will enter heart through the so-called "pulmonary pit", arrowed, which at this stage is flanked by prominent right and left pulmonary ridges. Picture B, at embryonic day 10.5 , when the embryo has 42 somites, shows the primary septum growing down towards the atrioventricular cushions. Its upper edge has broken down to form the secondary foramen. Note that the left superior caval vein, now formed from the left cardinal vein, is a discrete structure within the left atrioventricular groove. Picture $\mathrm{C}$, again from an embryo of 10.5 days, but now with 45 somites, shows the pulmonary vein opening as a solitary channel inferiorly to the left atrium, the systemic venous sinus, enclosed by the venous valves, now having become incorporated into the right atrium. The right pulmonary ridge has now expanded to become the vestibular spine (asterisk).

chamber, and fuses with the atrioventricular endocardial cushions, the primary atrial septum interposes between the right sided systemic venous component and the orifice of the pulmonary vein (fig 2). At this stage, the 16th horizon within the Carnegie system, ${ }^{12}$ the pulmonary vein is a solitary channel at its junction with the primary atrium, with its mouth positioned posteriorly, inferiorly, and to the left of the vestibular spine (fig 4). Accompanying the growth of the primary septum into the atrium, there has been expansion of the vestibular spine, by now covered by its own mesenchymal cap, with additional tissues entering the heart from the body wall through the spine (fig 2C). It is the expansion of the spine which unifies the mesenchymal cap on the primary atrial septum with the fused atrioventricular cushions, obliterating the primary atrial foramen in the process, and forming the basis of the fibrous septal structures of the heart (fig 5 ).

Before closure of the primary foramen, the upper edge of the primary septum, close to its site of origin from the atrial roof, breaks down to form the secondary interatrial communication (figs 3 and 5). Such establishment of a secondary foramen is essential if the systemic venous return is to

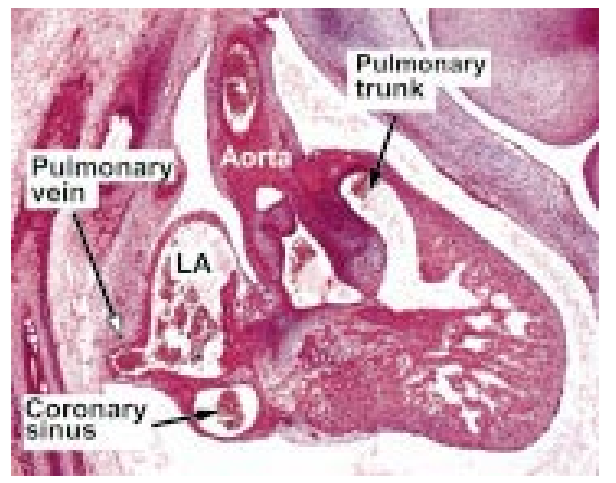

Figure 4 This section, taken in the sagittal plane from a human embryo at the 20th Carnegie stage, shows the solitary pulmonary vein entering inferiorly to the left atrium. Only with subsequent growth do four pulmonary veins enter that atrial roof, but this process is necessary to produce the so-called "septum secundum", in reality the infolded atrial roof (see fig 6). continue to reach the left side of the heart during the remainder of fetal development. Within this period, the atrioventricular canal has also expanded rightward, establishing the connection between the newly formed morphologically right atrium and the distal part of the ventricular loop, which has now become the right ventricle. ${ }^{13}$ The musculature of the atrioventricular canal itself becomes incorporated into the atrial chambers as the vestibules of the atrioventricular valves. Forward growth of the vestibular spine, binding the base of

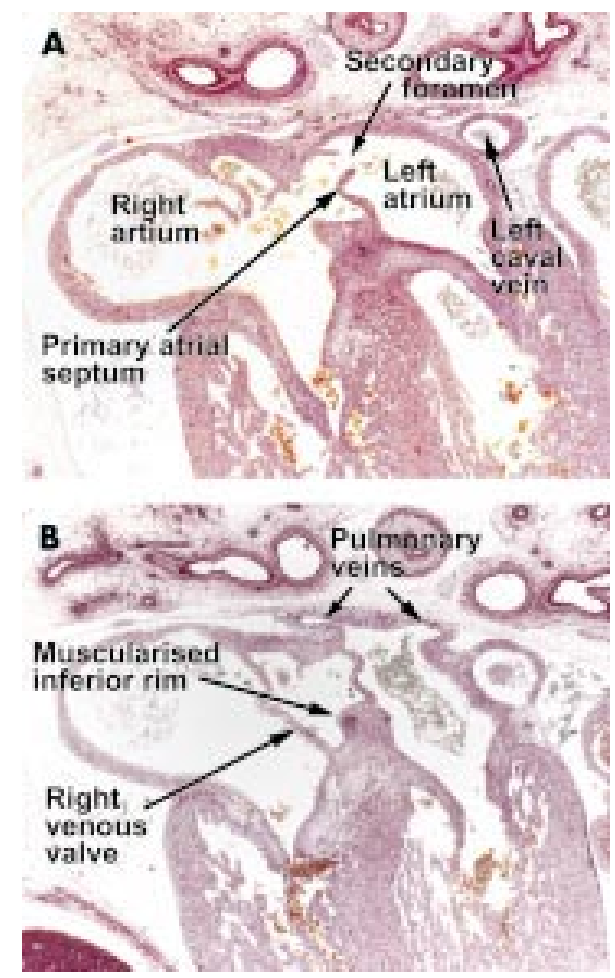

Figure 5 These sections are from a human embryo at Carnegie stage 20/21, showing the beginnings of the infolding of the atrial wall which will produce the rims of the oval fossa. Section $A$ is taken cranial to section $B$. Note the ongoing muscularisation of the antero-inferior rim of the oval fossa (see also fig 10). 


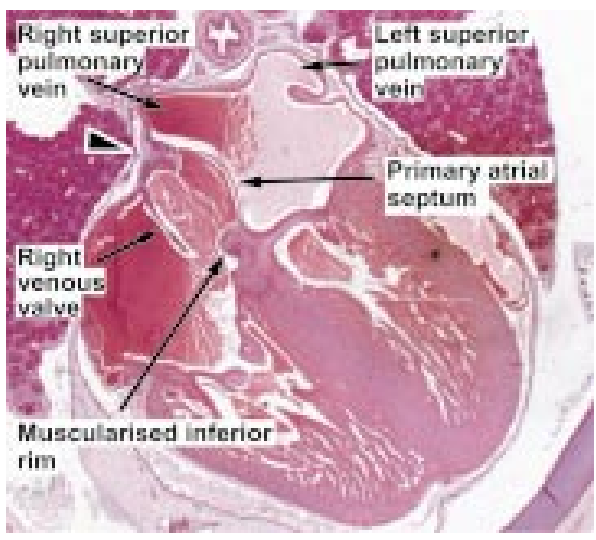

Figure 6 This section, from a human embryo at 11 weeks of development, shows the continuing infolding of the atrial roof as the pulmonary veins become incorporated into the left atrium.

the primary septum to the upper surface of the fused atrioventricular cushions, has also carried forward the inferior ends of the valves of the systemic venous sinus (fig 5). The expanded vestibular spine itself then becomes muscularised to form a bulbous structure that reinforces the base of the primary atrial septum. The thinner upper margin of the primary septum itself persists as the flap valve of the oval foramen. At this stage, representing the 21st stage of the Carnegie horizons, the pulmonary vein continues to drain inferiorly to the left atrium, but has divided into its right and left branches. ${ }^{12}$ As yet, there is no formation of a "secondary" septum within the roof of the dividing atrial chambers adjacent to the mouth of the superior caval vein, although evidence of infolding has begun to appear more inferiorly (fig 5).

Only subsequent to the eighth week of development does the initially solitary pulmonary vein begin fully to become incorporated into the body of the primary atrium, itself now largely part of the developing left atrium. By the 12th week of

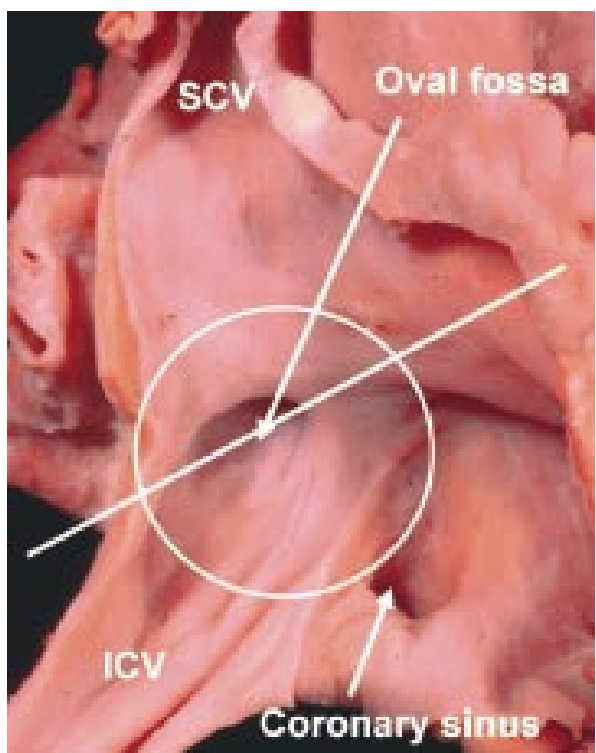

Figure 7 This picture of the human right atrium, taken from posteriorly and the right, shows how, at first sight, an extensive septal area, within the circle, separates the right from the left atrium. The true situation is shown in fig 8 , which is a cross section along the line shown in the figure. SCV, superior caval vein; ICV, inferior caval vein. development, the superior right sided pulmonary vein has become a separate tributary of the left atrium. Concomitant with this change, the atrial roof has infolded adjacent to the mouth of the superior caval vein to form the antero-superior margin of the oval foramen. ${ }^{14}$ This process of infolding, when complete (fig 6), provides the buttress against which the flap valve can close in postnatal life. Concomitant with incorporation of the pulmonary veins to form the extensive posterior left atrial wall, the two atrioventricular junctions, now discrete and separate structures incorporating the insulating tissues of the atrioventricular grooves, have expanded posteriorly and inferiorly. The expansion of the right atrial wall relative to the base of the ventricular mass sandwiches the inferior atrioventricular groove as a layer of fibroadipose tissue between the atrial and ventricular musculatures in the floor of the triangle of Koch. ${ }^{15}$

Thus, subsequent to the completion of septation, the definitive atriums each possess a part of the body of the primary atrium, an appendage, a vestibule, and a venous component. They remain in continuity with each other through the oval foramen. The newly muscularised antero-inferior margin of the oval foramen, derived from the vestibular spine, is anchored to the fibrous skeleton, itself formed from the atrioventricular cushions. The primary atrial septum persists as the flap valve of the oval foramen. The antero-superior rim, against which the flap valve abuts, and which is usually described as the "septum secundum", is the infolding now existing between the junction of the superior caval vein to the right atrium and the right pulmonary veins to the left atrium.

\section{DEFINITIVE STRUCTURE OF THE ATRIAL SEPTUM}

Concepts of development, if correct, must provide the basis for understanding definitive atrial structure. When the right atrium is opened parietally, there is, at first sight, an extensive area of potential communication with the cavity of the left atrium (fig 7). Sectioning across this area (fig 8), however, shows that only a small part is a septal structure, when a septum is defined as that part which can be removed without exiting from the cavities of the heart. ${ }^{16}$ The parts that can be removed to provide an interatrial communication are the floor of the oval fossa, the flap valve, and the part of the antero-inferior rim of the fossa which abuts on the vestibule of the tricuspid valve (fig 9). The floor of the oval fossa represents the embryonic primary atrial septum, while the anteroinferior margin of the fossa has been produced by muscularisation of the vestibular spine (fig 10). The extensive area

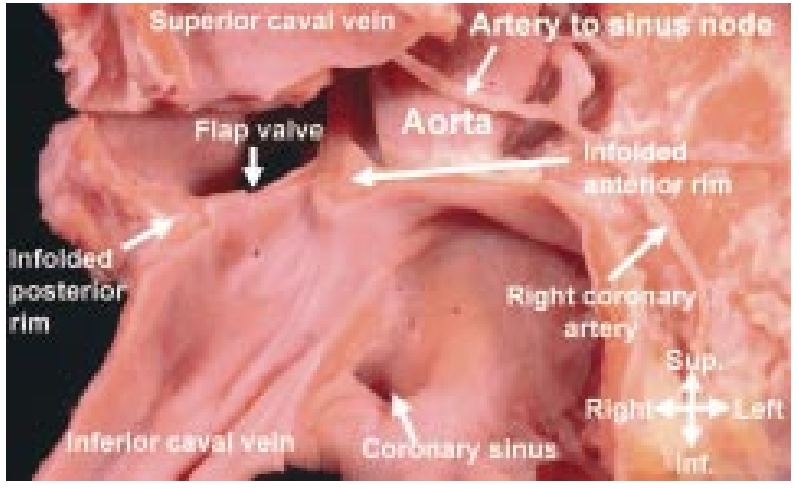

Figure 8 This section, along the line shown in fig 7, reveals how the rims of the oval fossa anteriorly and posteriorly are folds of the atrial wall. Note the relation of the anterior atrial wall to the aortic root. 


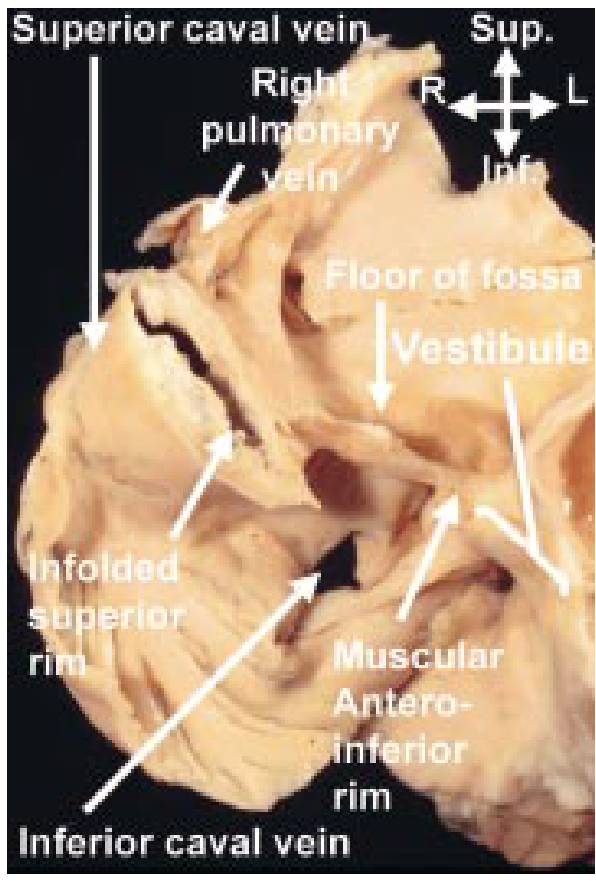

Figure 9 This section, taken in "four chamber" plane through an adult human heart, shows how the so-called "septum secundum" is a deep infolding between the connections of the pulmonary veins to the left atrium, and the superior caval vein to the right atrium. The "compass" shows the orientation. Sup, superior; Inf, inferior; R, right; L, left.

extending between the superior margin of the oval fossa and the mouth of the superior caval vein, although usually described as the "septum secundum", is a deep infolding of the atrial wall. This infolding, which is positioned between the connections of the systemic venous tributaries to the right atrium and the right pulmonary veins to the left atrium, is filled with extracardiac adipose tissue (figs 9 and 10). The flap valve of the septum, a fibromuscular structure in adult life, abuts against the fold to close the oval foramen. As long as the flap valve is of greater dimensions than the floor of the oval
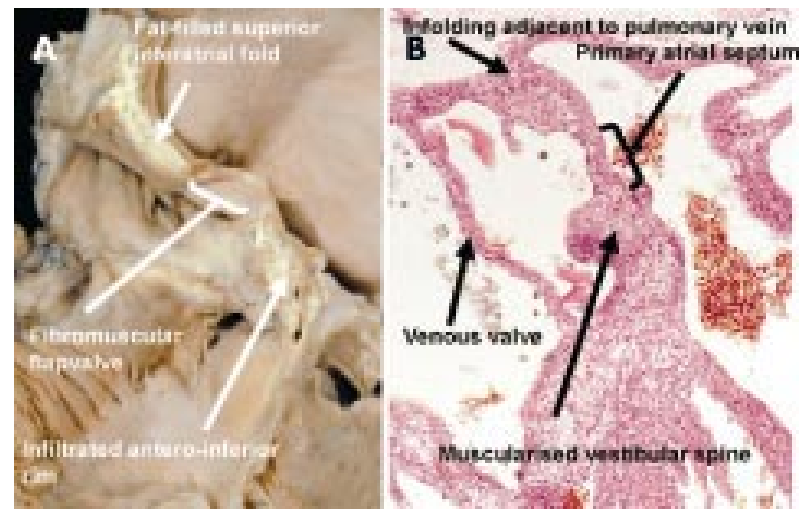

Figure 10 The left panel shows another "four chamber" section through an adult human heart. It shows not only the accumulation of fat within the superior interatrial fold, but also the fat which is infiltrating through the inferior atrioventricular groove into the inferior rim of the oval fossa. The right panel shows a section from a human embryo of 7-8 weeks' development, when two pulmonary veins have been incorporated into the left atrium. It shows the muscularisation of the vestibular spine, which forms the inferior rim of the oval fossa.
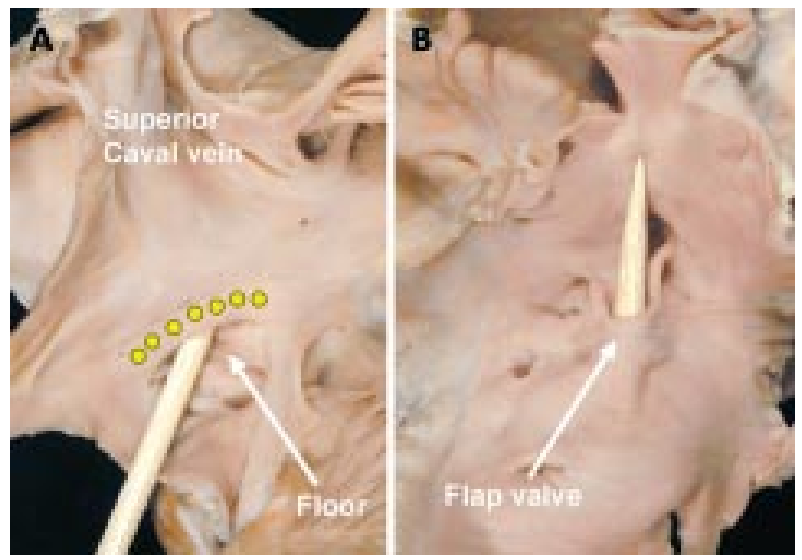

Figure 11 The pictures show a probe-patent oval foramen in a human heart, viewed from the right (left panel) and left (right panel) atrial aspects. The probe is placed through the foramen, separating the flap valve in the left atrium from the rims of the oval fossa (yellow dots).

fossa, and the left atrial pressure exceeds that in the right atrium, there will be no potential for interatrial shunting even if the two structures are not mechanically fused. This is of major clinical significance, since such anatomic fusion has occurred only in between two thirds and three quarters of individuals, even in the eighth or ninth decades of life. ${ }^{17}$ Thus, in that minority in which fusion has not occurred, or in other words, those with a probe-patent oval foramen (fig 11), there is the potential for interatrial shunting should right atrial pressure exceed left, and hence the potential for paradoxical embolism. The true atrial septum, therefore, is made up of the flap valve of the oval foramen and its bulbous antero-inferior base.

Antero-superiorly, the right atrial wall itself directly overlies the aortic root (fig 9). The bulbous antero-inferior margin is perforated by the tendon of Todaro, this being the extension of the inferior junction of the valves of the systemic venous sinus. The valves themselves regress to greater or lesser extent in the postnatal heart. The atrial wall itself continues anteriorly beyond the bulbous inferior rim of the oval foramen as the smooth vestibule of the tricuspid valve, which superiorly forms the border of the atrioventricular membranous septum, an integral part of the central fibrous body. In the definitive heart, this fibrous area has become incorporated into the aortic root. It is perforated by the atrioventricular conduction axis as it passes from the atrioventricular node to become the bundle of His (fig 12). This transition from node to bundle marks the initial position of that part of the embryonic atrioventricular canal musculature which persisted as the atrioventricular bundle, the conduction tissue itself being a specialised part of the canal musculature. ${ }^{18}$

The extensive vestibule of the tricuspid valve seen inferiorly is derived from expansion of the right atrioventricular junction subsequent to the completion of embryonic septation. This is confirmed by the extent of the fibroadipose tissue to be found in the inferior atrioventricular groove between the atrial and ventricular musculatures. As already discussed, this incorporation of fibroadipose tissue between layers of myocardium produces the muscular atrioventricular sandwich, ${ }^{15}$ an area which we previously considered, incorrectly, to represent a true muscular septal structure. ${ }^{19}$ Also opening into this inferior corner of the right atrium is the mouth of the coronary sinus, a remnant of the embryonic left sinus horn. The mouths 


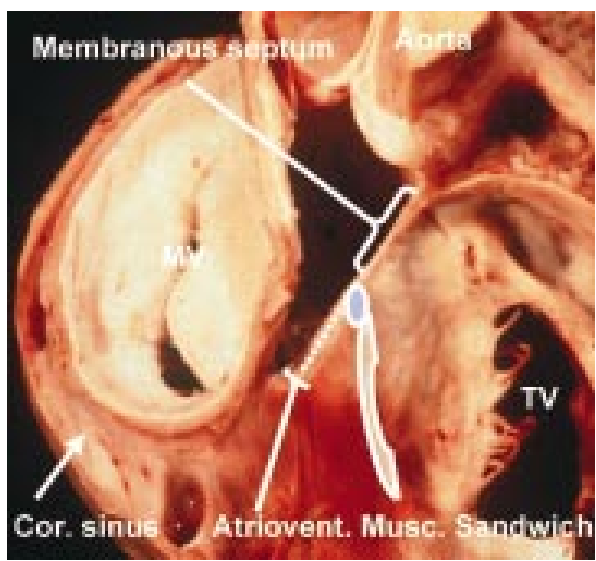

Figure 12 This dissection of a human heart shows the relations between the right and left atrioventricular junctions and the subaortic outflow tract. It has been made by removing the atrial walls, along with the non-coronary sinus of the aortic root. It shows the location of the membranous septum, which is penetrated by the atrioventricular conduction axis as it passes from the atrioventricular node (blue oval) to reach the crest of the muscular ventricular septum. The dots show the tissues of the inferior atrioventricular groove which interpose between the atrial and ventricular layers of the muscular atrioventricular sandwich. The red cross hatched area is the vestibule of the tricuspid valve (TV). MV, mitral valve; Cor sinus, coronary sinus.

of the inferior and superior caval veins represent the remaining tributaries of the right sinus horn, with the prominent terminal crest and the remnants of the right venous valve marking their boundary with that part of the right atrium derived from the primary atrial component of the heart tube. Most of this primary atrium is made up of the pectinated atrial appendage. The so-called "sinus septum" is no more than the fold of the atrial walls between the mouths of the inferior caval vein and the coronary sinus.

The location of the flap valve of the septum is much better appreciated when viewed from the left side, with two horns anchoring the flap to the left side of the infolded superior rim, albeit often without anatomic fusion (fig 11). The left atrium has its own appendage, with its pectinated walls, positioned anteriorly and superiorly. The posterior wall is exclusively smooth walled, being derived by incorporation of the extensive pulmonary venous component between the vestibule and the body of the atrium, the latter forming the extensive atrial dome.

\section{COMMENT}

It was HL Mencken who pointed out that, for every complex question, there was a simple and straightforward answer, which was usually wrong! This is certainly the case with the development of the atrial septum. There is no evidence of which we are aware to substantiate the notion that primary and secondary septums grow into the developing atrium, becoming perforate and overlapping so as to produce the definitive oval foramen. It is certainly the case that the primary septum grows into the primary atrial component of the heart tube, and that it persists as the flap valve of the definitive oval foramen. The bulbous muscular antero-inferior rim of the septum, in contrast, has a much more complex mode of development. It represents muscularisation of an area of mesenchyme which grows into the heart. The mesenchyme is derived from the cap that is carried on the leading edge of the primary septum, along with the mass known as the vestibular spine. Intriguingly, the mechanism of muscularisation of this population of cells, which grow into the heart at the venous pole, has much in common with similar muscularisation recently shown to be involved with transformation of the ingrowth of cells through the aortopulmonary septum at the arterial pole of the heart. ${ }^{20}$ Be that as it may, this part of the septum can reasonably be equated with the inferior part of the "septum secundum" depicted in standard textbooks. The position of this septal component, located as it is between the vestibules of the mitral and tricuspid valves, may well explain why His named its primordium as the "spina vestibuli". ${ }^{5}$ If this bulbous base of the septum can justifiably be considered to be a secondary septal structure, the deeply infolded antero-superior rim certainly cannot. And this is the area most usually depicted as the "septum secundum" in classical texts! Lipomas in this area are usually held to involve this "secondary" septum. ${ }^{3}$

Much of any disagreement regarding this issue, of course, depends on the definition used for a "septum". As we have explained, we define a septum as a structure that can be removed without exiting from the cavities of the heart. ${ }^{16}$ The distinction between structures fulfilling this definition, and those folds or sandwiches which can similarly interpose between cardiac cavities, is important to the clinician working in the heart, be his or her discipline surgery or interventional catheterisation. The surgeon needs to be aware that, if cuts are made through a fold or sandwich, they pass outside the heart. The congenital cardiac surgeon is certainly aware that incisions through the deep superior interatrial groove offer a good access to the left atrium. In the days of surgical procedures producing atrial redirection, it was equally well established that dissections of the walls of the groove provided increased lengths of atrial wall for use in the subsequent reconstruction. Nowadays, the interventional cardiologist is well aware of the difference in structure between the firm antero-inferior base of the atrial septum, which provides a firm foundation for anchorage of devices used for interventional closure, as opposed to the superior rim, which can often be effaced. ${ }^{9}$ It is our hope that, in this review, we have shown how these important clinical differences between septums, flaps, folds, and sandwiches are well explained once it is established properly how the atrial chambers develop.

\section{Authors' affiliations}

R H Anderson, Cardiac Unit, Institute of Child Health, London, UK N A Brown, S Webb, Department of Anatomy and Developmental Biology, St Georges Hospital Medical School, London, UK

\section{REFERENCES}

1 Röse C. Zur Entwicklungsgeschichte des Saugerthierherzens. Morphol Jahr 1899;15:436-56.

2 Christie GA. The development of the limbus fossae ovalis in the human heart: a new septum. J Anat 1963;97:45-54.

- These works show that it has long been recognised that the so-called "septum secundum" is, in reality, no more than an infolding between the connections of the pulmonary veins to the left atrium, and the caval veins to the right atrium.

3 Basso C, Barbazza R, Thiene G. Images in cardiovascular medicine. Lipomatous hypertrophy of the atrial septum. Circulation 1998;97:1423.

- Although the authors claim that their image demonstrates the collection of fat within the atrial septum, in fact it is clear that the fat has accumulated within the superior interatrial groove.

$4 \mathrm{Li} \mathrm{J}, \mathrm{Ho}$ SY, Becker AE, et al. Multiple cardiac lipomas and sudden death - a case report and literature review. Cardiovasc Pathol 1998;7:349-52.

- The authors review an amazing heart which shows multiple lipomas, and show how the fat accumulates within the grooves of the heart, albeit that there is also infiltration of the myocardium. 
5 His W. Die Area interposita, die Eustachi'sche Klappe und die Spina vestibuli. In: Anatomie Menschlicher Embryonen. Leipzig: von FCW Vogel, 1880:49-152.

- The initial description of the "spina vestibuli". His's reconstructions are remarkably accurate, although he does not specify the nature of the vestibule in which the spine is formed.

6 Webb S, Brown NA, Wessels A, et al. Development of the murine pulmonary vein and its relationship to the embryonic venous sinus. Anat Rec 1998;250:325-34.

110 An experimental study of the mouse heart, using scanning electron microscopy, showing that the pulmonary vein canalises in the so-called "dorsal mesocardium", and not originating, as has been claimed, from the systemic venous sinus.

7 Webb S, Brown NA, Anderson RH. Formation of the atrioventricular septal structures in the normal mouse. Circ Res 1998:82:645-56.

- An extension of the work published in reference 6, emphasising that several primordiums are needed to achieve appropriate separation of the right and left atriums along with their atrioventricular junctions.

8 Anderson RH, Webb S, Brown NA. Clinical anatomy of the atrial septum with reference to its developmental components. Clin Anat 1999; 12:362-74.

- A review of the structure of the atrial septum, emphasising that the so-called "septum secundum" is no more than an infolding of the atrial roof.

9 Ferreira Martins JD, Anderson RH. Anatomy of the interatrial communication - what does the interventionist need to know? Cardiol Young 2000;10:464-73

- A review of atrial septal structure in the light of the increasing use of devices inserted on catheters to close holes within the oval fossa.

10 de la Cruz MV, Sanchez-Gomez C, Palomino MA. The primitive cardiac regions in the straight tube heart (stage 9) and their anatomical expression in the mature heart: an experimental study in the chick embryo. J Anat 1989;165:121-31.

11 Kelly RG, Brown NA, Buckingham ME. The arterial pole of the mouse heart forms from Fgf-10 expressing cells in pharyngeal mesoderm. Developmental Cell 2001;1:1-20.

- Two studies which question the classical concept that the heart develops as a series of segments within an initially straight primary heart tube. In fact, at no stage in normal development is there such a straight tube with venous, atrial, ventricular, and arterial components.

12 O'Rahilly R, Müller F. Developmental stages in human embryos. Washington: Carnegie Institution of Washington Publication 637, 1987.

- The classical study which provided the landmarks for categorising the stages of development of the human embryo.

13 Lamers WH, Wessels A, Verbeek FJ, et al. New findings concerning ventricular septation in the human heart. Circulation 1992;86:1 194-205.
A study which used an antibody to the nodose ganglion of the chick to chart the development of the atrioventricular junctions of the human heart, showing that the right ventricle was formed exclusively from the distal component of the ventricular loop.

14 Webb S, Kanani M, Anderson RH, et al. Development of the human pulmonary vein and its incorporation in the morphologically left atrium. Cardiol Young 2001;11:632-42.

- An examination of serial sectioned human embryos which showed that, as in the mouse, the pulmonary vein canalised within the dorsal mesocardium. No evidence was found to support the concept that the vein originated from the systemic venous sinus. The study showed that the subsequent incorporation of four pulmonary veins to the atrial roof was a relatively late event during development.

15 Anderson RH, Ho SY, Becker AE. Anatomy of the human atrioventricular junctions revisited. Anat Rec 2000;260: 81-91.

16 Anderson RH, Brown NA. The anatomy of the heart revisited. Anat Rec 1996;246:1-7.

These two studies questioned the definitions used for septal structures, emphasising that true septums are the walls of the heart which can be removed without exiting from the cardiac cavities. The first study also reviewed the relationships of the conduction axis to the atrioventricular junctions.

17 Hagen PT, Scholz DG, Edwards WD. Incidence and size of patent foramen ovale during the first 10 decades of life: an autopsy study of 965 normal hearts. Proc Staff Meet Mayo Clin 1984;59:1489-94.

- An important study which showed that, irrespective of age, about one third of the population retains probe patency of the oval foramen.

$18 \mathrm{Kim}$ J-S Viragh Sz, Moorman AFM, et al. Development of the myocardium of the atrioventricular canal and the vestibular spine in the human heart. Circ Res 2001;88:395-402.

- A study of the development of the human heart, showing how the vestibular spine becomes muscularised to form the antero-inferior margin of the oval foramen.

19 Becker AE, Anderson RH. What's in a name? J Thorac Cardiovasc Surg 1982;83:461-9

- The initial study which emphasised that a common atrioventricular junction was the pathognomonic feature of hearts with so-called "atrioventricular canal malformations". The study also showed that the hole between the four cardiac chambers was, in reality, an atrioventricular septal defect.

20 van den Hoff MJ, Moorman AFM, Ruijter JM, et al. Myocardialization of the cardiac outflow tract. Dev Biol 1999;212: 477-490.

- The embryological study which had first demonstrated the importance of myocardialisation of the endocardial cushions, this investigation concentrating of the formation of the outflow segments. 